\title{
Gleichgewichtsorgans: Gerätetechnische Realisierung für den Einsatz in der Praxis
}

\author{
Caloric irrigation of the vestibular organ using near infrared: A device for \\ use in clinical practice
}

L.E. Walther

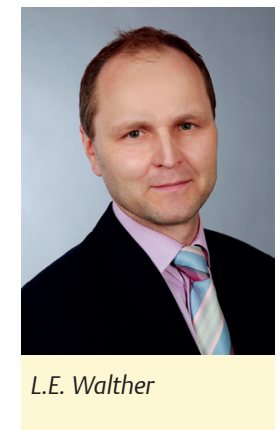

\section{Background}

Irrigation of the vestibular organ for diagnostic purposes can be performed by using different caloric stimuli. Apart from traditional stimulation by the use of water, dry and wet air can be applied. Furthermore, infrared (IR) radiation provides an effective warm stimulus of the vestibular organ. A device for IR based irrigation of the equilibrium organ via the external auditory canal is presented, which is useful for further use in clinical practice. IR radiation as a caloric stimulus combines a number of advantages: The stimulus can be easily applied and is quiet. Contrary to stimulation with warm dry air, the IR stimulus is more effective. A paradoxical nystagmus does not occur. During application, no arousal effects or unpleasant feeling can be observed. The warm stimulus can be applied by different stimulus modalities such as continuous stimulation with different intensities and pulsating stimulation. This allows to apply a warm stimulus according individual anatomical situations such as according to individual chronic otitis media (ear drum defects), inflammation of the external auditory canal, chronic mastoid cavities, and conditions in which individuals show hypersensitivity to water stimulation. Moreover, the method can be used also in normal individuals. schiedlichen Intensitäten und „Puls“-Modus. Diese Steuerbarkeit des Stimulus erlaubt eine Anpassung an individuelle Gegebenheiten. So kann die IR-Reizung z.B. bei speziellen anatomischen Situationen, wie bei chronischen Mittelohrentzündungen (Trommelfellperforation), Entzündungen der Ohrregion, nach Ohrradikaloperationen mit Anlage einer offenen Mastoidhöhle und subjektiver Überempfindlichkeit bei anderen thermischen Reizen eingesetzt werden. Darüber hinaus spricht nichts gegen einen Einsatz als Screeningmethode im klinischen Alltag. 


\section{Einleitung und Grundlagen} $\nabla$

Die drei Bogengänge, insbesondere die lateralen Bogengänge, sowie der Nervus vestibularis, sind mit ihren Sinneseingängen in der Lage, Drehbeschleunigungsreize aus der Umwelt sensorisch aufzunehmen und weiterzuleiten (vestibulookulärer Reflex, VOR). Damit wird, gemeinsam mit anderen sensorischen Systemen, eine störungsfreie und somit für den menschlichen Organismus unter gesunden Bedingungen nicht realisierbarer Beitrag zur Orientierung im Raum bei den Anforderungen im Alltag aber auch bei höheren Beanspruchungen gewährleistet.

Störungen des VOR führen zu einer Störung der Orientierung im Raum. Symptome sind unter anderem Blickunschärfen bei Kopf-Körperbewegungen, die individuell unterschiedlich als „Schwindel“ interpretiert werden.

Für die Differentialdiagnostik von Störungen des VOR stehen heute mehrere Methoden zur Verfügung. Aufgrund Ihrer Objektivität und Seitenspezifität zählen der Video-Kopfimpulstest und die thermische Prüfung zu den wichtigsten Untersuchungsverfahren. Sie reflektieren Störungen des VOR im geringen (thermische Prüfung) und höheren Frequenzbereich (Video-Kopfimpulstest). Beide Methoden sind für eine exakte Aussage über das Störungsmuster bei peripheren Vestibulopathien notwendig.

In der klinischen Praxis werden hauptsächlich Wasser und Luft als Reizmedien eingesetzt, um eine Aussage über die thermische Erregbarkeit zu erhalten. Einen internationalen Konsens über den Einsatz dieser Methoden gibt es nicht, ebenso fehlen evidenzbasierte Untersuchungen. Während Wasser nur bei intakten und reizlosen Verhältnissen im Gehörgang verwendet werden kann, muss bei großen Trommelfelldefekten oder Entzündungen mit Luft gereizt werden. Auf Grund der niedrigen Wärmekapazität wird Luft beispielsweise bei der Funktionsprüfung exponierter Gleichgewichtsorgane (Radikalhöhle eines Ohres) verwendet.

Bei diesen Patienten kann Schwindel schneller entstehen und intensiver sein, denn bereits geringe Temperaturunterschiede in der Nähe der Bogengänge genügen, um einen Nystagmus (VOR) zu generieren.

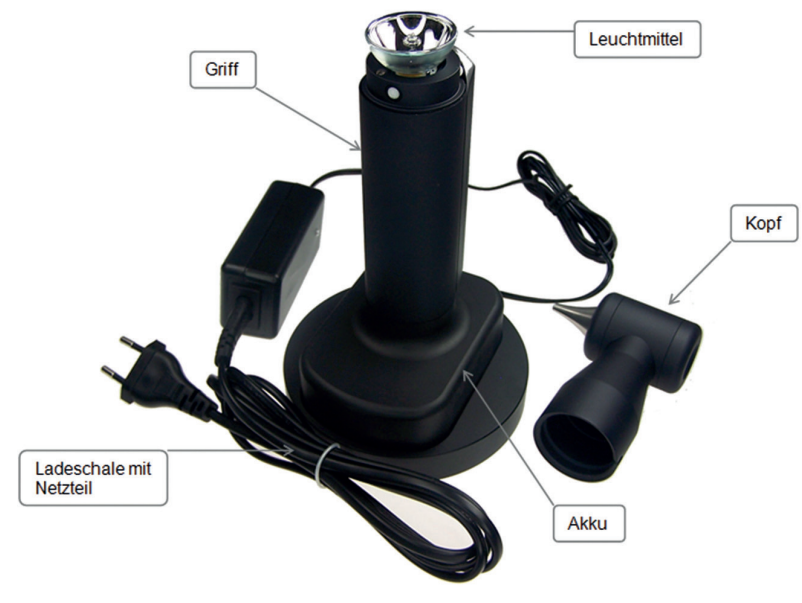

Abb. 1: Baugruppen des IR-Reizgerätes.
Ein Luftreiz lässt sich im Gegensatz zu Wasser schlechter dosieren und ist mit einer Geräuschentwicklung im Gehörgang verbunden. Bei der Reizung mit Raumluft (44 Grad) kann unter Umständen Verdunstungskälte zur Entstehung eines paradoxen Nystagmus führen.

Die Nahinfrarotreizung stellt eine weitere Möglichkeit dar, um eine Aussage über den durch Warmreiz induzierten VOR zu erhalten.

\section{Grundlagen der Nahinfrarotrei- zung des Gleichgewichtsorgans \\ $\nabla$}

Sichtbares Licht wird in einem Wellenlängenbereich von menschlichen Auge 400 bis $760 \mathrm{~nm}$ wahrgenommen. Für das Auge unsichtbares Nahinfrarot (Wärmestrahlung) hingegen wird in einem Wellenlängenbereich von ca. 760 nm bis $1400 \mathrm{~nm}$ emittiert. Die Ausbreitung der nah-infraroten Strahlung erfolgt mit Lichtgeschwindigkeit. Ein Energieeintrag, z. B. in den Gehörgang, führt in Abhängigkeit von der Wellenlänge und der optischen Strahlungsleistung zu einer Erwärmung der Haut des äußeren Gehörgangs, die als inhomogenes Medium eine Grenzschicht darstellt. Beim Auftreffen auf die Haut kommen Wechselwirkungen zustande. Dadurch werden Effekte wie Reflexion, Streuung und Absorption induziert. Die besondere Eigenschaft von Nahinfrarotstrahlung ist ihre große Eindringtiefe in die Haut. Bei 900 bis 1000 nm erreicht diese „Tiefenwirkung“ ihr Maximum und beträgt je nach optischer Strahlungsleistung und Art der Strahlungsquelle mehrere Millimeter. Dieser Effekt kann bei der Reizung des Gleichgewichtsorgans mit monochromatischen und breitbandigen
Lichtquellen (mit relevantem Nahinfrarot und sichtbarem Lichtanteil) ausgenutzt werden (sogenanntes „diagnostisches Fenster"). Somit ist Nahinfrarotstrahlung hervorragend geeignet, Wärmemengen effektiv und schonend in den Organismus einzutragen, was bei der thermischen Reizung genutzt werden kann.

Die Methode der Nahinfrarotreizung wurde bisher in mehreren Studien bei Patienten mit normalen und pathologischen Gehörgansverhältnissen untersucht. (Walther LE et al. Laryngorhinootologie. 2003; 82: 687-92; Walther LE et al. Laryngorhinootologie. 2004; 83: 88-95; Walther LE et al. HNO. 2004; 52: 525-32; Walther LE et al. Acta Otorhinolaryngol Ital. 2011; 31: 90-5; Ferrè ER et al. PLoS One. 2015 Apr 13; 10(4):e0124573. doi: 10.1371/journal. pone.0124573. eCollection 2015).

\section{Vorteile der Nahinfrarotreizung und Indikationen $\nabla$}

Die Reizapplikation erfolgt bei einer Nahinfrarotreizung lautlos. Da es sich um Strahlungsenergie handelt, ist das Reizmedium „steril“. Im Vergleich zur Stimulation mit warmer trockener Luft sind die induzierten auswertbaren Nystagmusparameter (Geschwindigkeit der langsamen Nystagmusphase, Nystagmusfrequenz) höher. Ein paradoxer Nystagmus infolge Verdunstungskälte, wie bei trockener warmer Luft, tritt bei der Nahinfrarotreizung nicht auf. Die Stimulation wird im Gegensatz zu allen anderen Methoden als angenehm empfunden oder nicht wahrgenommen. Der applizierte Reiz ist steuerbar (Dauer- und Impulsapplikation) und flutet relativ langsam an. 
Die Nahinfrarotreizung kann bei speziellen anatomischen Situationen, wie bei chronischen Mittelohrentzündungen (Trommelfellperforationen), Entzündungen der Ohrregion, nach Ohrradikaloperationen mit Anlage einer offenen Mastoidhöhle und subjektiver Überempfindlichkeit bei anderen thermischen Reizen und somit auch bei normalen Verhältnissen im Gehörgang eingesetzt werden. Sie stellt damit eine Ergänzung zu den existierenden Verfahren bei der Prüfung auf Warmreiz dar.

\section{Gerätebeschreibung}

Das Nahinfrarot-Reizgerät besteht aus einem handlichen Gerät mit austauschbaren Baugruppen. Ein Bedienfeld mit LEDLeuchten gestattet eine wahlweise Einstellung des Stimulus (Dauer- bzw. Impulsmodus mit unterschiedlichen Reizzeiten und Intensitäten). Der abnehmbare Kopfteil mit Ohrtrichter gestattet einen Einblick in den Gehörgang wie bei einem Otoskop. Als Quelle für die Infrarotstrahlung dient eine speziell hergestellte Halogenlampe. Sie ist für die Einkopplung von

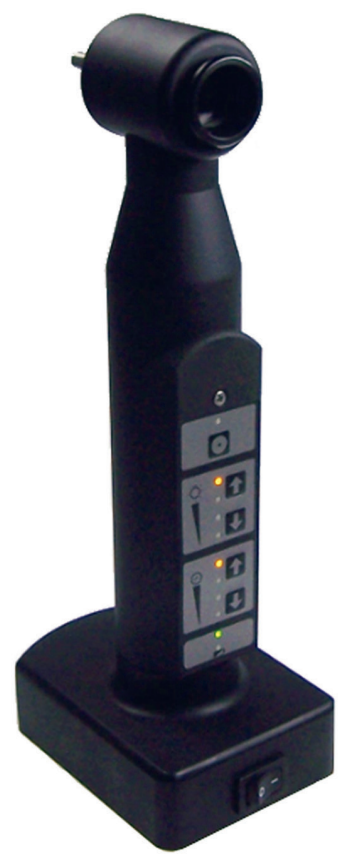

Abb. 2: Bedienfeld des IR-Reizgerätes.
IR-Strahlung optimiert und deckt den Wellenlängenbereich des diagnostischen Fensters (900 -1000nm) effektiv ab. Das Nahinfrarot-Reizgerät funktioniert mit einem aufladbaren Akku und steht in einer Ladeschale mit Netzteil, über welche der Akku geladen wird. Das Gesamtgewicht des Gerätes beträgt ca. $700 \mathrm{~g}$.

\section{Fazit für die Praxis \\ $\nabla$}

Die vorgestellte gerätetechnische Realisierung der Methode der Nahinfrarotreizung des Gleichgewichtsorgans eignet sich für den Einsatz in der Praxis. Vorteile der Warmreizung mit Nahinfrarot ist die lautlose und „sterile“ Reizapplikation. Die IR Stimulation ist der Reizung mit Raumluft überlegen. Sie ist vor allem bei speziellen anatomischen Situationen, wie bei chronischer Otitis media, Entzündungen der Ohrregion, nach Ohrradikaloperationen mit Anlage einer offenen Mastoidhöhle und subjektiver Überempfindlichkeit bei anderen thermischen Reizen aber auch bei normalen Verhältnissen im äußeren Gehörgang, einsetzbar.

\section{Danksagung}

Herrn Eckart Rogge, Berlin, wird für die technische Beratung bei der Abfassung dieses Artikels gedankt.

Interessenkonflikt: Der korrespondierende Autor gibt keine Interessenkonflikte an.

Korrezpondenzadresse

Prof. Dr. med. habil. Leif Erik Walther

HNO-Gemeinschaftspraxis

Main-Taunus Zentrum

65843 Sulzbach (Taunus)

Telefon: +49 (069) 309905

Telefax: +49 (069) 3089096

E-Mail: Leif.Walther@hno-praxis-sulzbach.de 